\title{
Assessment of Brain Tumor Displacements after Skull-based Registration: A CT/MRI Fusion Study
}

Qianyi Xu*, George Hanna, Yongjun Zhai, Sucha Asbell, Jiajin Fan, Tamara LaCouture, Yan Chen, Leonard Kim and Gregory Kubicek

Department of Radiation Oncology, MD Anderson Cancer Center, Cooper Mount Laurel, USA

*Corresponding author: Qianyi Xu, Ph.D., Department of Radiation Oncology, MD Anderson Cancer Center, Cooper Mount Laurel, NJ 08054, USA, Tel: 520-331-9966; E-mail: xuqianyi@gmail.com

Received date: Sep 30, 2015, Accepted date: Nov 07, 2015, Publication date: Nov 10, 2015

Copyright: (C) $2015 \mathrm{Xu}$ Q, et al. This is an open-access article distributed under the terms of the Creative Commons Attribution License; which permits unrestricted use; distribution; and reproduction in any medium; provided the original author and source are credited.

\begin{abstract}
Purpose: To assess brain tumor displacements between skull based and soft-tissue based matching during CTMRI fusion for a total of 35 brain lesions.

Methods: Twenty-five patients who underwent CT and MRI scans in the same day were retrospectively recruited into the study. Semi-automatic skull based fusion was first performed and reviewed on a Treatment Planning System (TPS). A secondary fine-tuning of the fusion was then performed, if mismatch was observed in the tumor or neighboring soft-tissue, using nearby visible soft-tissue, such as gyri, sulci, and fissures. Two physicists fine-tuned the secondary fusion until the best match could be agreed upon. The resulting rotations and translations between the two fusions were recorded, which indicated local displacements between skull based and soft-tissue based matching. We further created a PTV by expanding a $2 \mathrm{~mm}$ margin around the GTV after skull-based fusion, and then evaluated the coverage of the GTV within the PTV after fine tuning with soft-tissue based fusion.
\end{abstract}

Results: In 29 of the 35 lesions, minor to no mismatch was found between the soft-tissue and skull based fusions. The corresponding translational and rotational shifts were $0.05 \pm 0.63 \mathrm{~mm}$ (LR), $0.01 \pm 0.79 \mathrm{~mm}$ (AP), 0.37 $\pm 1.01 \mathrm{~mm}$ (SI); $-0.15 \pm 0.67^{\circ}$ (pitch), $-0.19 \pm 0.34^{\circ}$ (yaw), and $-0.12 \pm 0.49^{\circ}$ (roll). Thus the GTV, after soft-tissue based fusion, was $100 \%$ covered by the PTV. However, in the remaining 6 lesions in the study, noticeable displacements were observed between the skull and soft-tissue based fusions. Excluding an outlier lesion, the mean translational and rotational shifts for 5 of the 6 remaining lesions were $0.90 \pm 2.15 \mathrm{~mm}$ (LR), $1.50 \pm 2.27 \mathrm{~mm}$ (AP), $-1.01 \pm 1.83 \mathrm{~mm}(\mathrm{SI}) ;-1.42 \pm 3.12^{\circ}$ (pitch), $0.02 \pm 0.83^{\circ}$ (yaw), and $-0.17 \pm 0.68^{\circ}$ (roll). For the outlier lesion, the GTV was nearly missed by the PTV, and for the rest of the 5 lesions, the mean coverage of the GTV was $98.9 \%$ within the PTV.

Conclusion: In a small portion of lesions, our study showed noticeable brain tumor displacement with typical patient setup in CT and MRI scans when using skull based fusion in comparison with soft-tissue fusion. Careful review of the skull based fusion is recommended by examining the match with nearby soft-tissue and/or tumors. If fusion deviations are found, it is also recommended to consider adding a margin to the GTV to account for such variations, since such variations could potentially affect target localization accuracy at the time of treatment.

Keywords: Tumor displacement; MRI; CT; Fusion; Motion; Skull

\section{Introduction}

Stereotactic radiosurgery (SRS) and stereotactic radiotherapy (SRT) deliver high doses to benign and malignant intracranial tumors in a single (SRS) to multiple (SRT) fractions. For patients with high risk of surgical complications, it provides a non-invasive alternative treatment to ensure local tumor control while sparing nearby critical structures. Reproducibility and accurate contouring of the tumor is of great importance since treatments consist of high doses delivered in a single or limited number of fractions. In the initial design of SRS and SRT, the skull was directly fixed to a frame to achieve high treatment precision [1]. Despite the invasive nature, the technique is still widely adopted in Gamma Knife treatments. In recent years, localization techniques have evolved into using non-invasive image guidance with similar treatment accuracy, including $\mathrm{kV}$ and $\mathrm{MV}$ cone beam computed tomography (CBCT) [2-5], optical systems [6-8], orthogonal x-ray imaging systems [9-11] and in-room CT [12]. With many of these systems a CT scan is used for treatment planning but an MRI scan is required to contour and define the target. Thus, overall accuracy depends on the ability to properly register or fuse the MRI scan with the planning CT scan.

Among all types of image guidance, the skull has been universally adopted as the image matching anatomy during SRS and SRT due to its rigidity and great visibility in all imaging modalities. This reliance on skull matching is common in multi-modality image fusions before treatment and in image guidance and target localization during treatment. This convention, however, relies on the assumptions that brain tumors keep the same relative position to the skull, and that the accuracy of matching simple skull geometries can pick up matching differences in complicated soft-tissue geometries and then can ultimately represent accurate tumor matching. Guckenberger et al. recently evaluated the reliability of the skull in SRS treatments of brain metastases and reported three-dimensional (3D) displacements of brain tumors between the skull and nearby soft-tissue [13]. In the study, 18 patients with intravenous (IV) contrast injection had an inroom CT scan followed by a CBCT scan before SRS their treatment. 
Page 2 of 6

The in-room CT and CBCT were fused to the planning CT based on skull and soft-tissue matching. The tumor displacement in each axis between these two fusions had high correlation $(r \geq 0.88)$. However, the tumor centroids between the two fusions revealed a mean $3 \mathrm{D}$ mismatch of $1.7 \pm 0.7 \mathrm{~mm}$ (maximum $2.8 \mathrm{~mm}$ ). Rotational shifts were not considered in the study. The mismatch indicates slight tumor-skull relative displacements between CT fusions.

In the field of neurological studies actual brain displacements have been the focus of research over the past decades since brain tissue motion is a biomechanical indication for most brain traumas. Various theoretical and experimental models have been developed to estimate brain motion relative to the skull when the brain was under mild impact and in static conditions (in different positions). In a typical design, the heads of volunteers moved on a short track and stopped by hitting a soft suspension. Meanwhile, MRI scans with high temporal resolution were acquired. The relative brain displacements $(2-5 \mathrm{~mm})$, as well as rotational shifts, were reported [14]. In another study, the volunteers had MRI scans of their brain while in different positions, and relative brain-skull motion was reported [15].

The setting in trauma studies is clearly different from a patient setting in SRS and SRT. However, the possibility of such motion is still insightful. We modified the work from Guckenberger et al. in our study by using MRI to take advantage of its superior soft-tissue contrast. After a skull-based fusion of the CT and MRI images, the alignment of nearby soft-tissue and tumors was reviewed. If any mismatch was observed, fine-tuning of the skull based fusion was performed until the best soft-tissue or tumor match was achieved. The two fusions were reviewed by two medical physicists (Y.Z. and Q.X.) and a radiation oncologist (G.K.), and the translational and rotational shifts between the two fusions were quantified. We further evaluated the coverage of the Gross Tumor Volume (GTV) by adding a small margin into an expanded Planning Target Volume (PTV) to account for such displacements of skull image guidance during treatment.

Many institutions use rigid skull registration for MRI and CT fusion, the subsequent SRS and SRT plans derived from this is potentially incorrect if skull fusion does not faithfully correspond to the soft tissue. The purpose of this project is to explore differences between skull based and soft tissue based fusion between CT and MRI and how this might affect SRS and SRT treatments.

\section{Methods and Materials}

\section{Patient and imaging settings}

Twenty-five previously treated patients with either primary or metastatic brain tumors were retrospectively recruited in this study. The patient recruitment criterion was the visibility of the tumor or nearby soft-tissues in the planning CT. The characteristics of the patients and tumors are summarized in Table 1. A total of 35 brain lesions were analyzed. All patients had a planning CT scan (LightSpeed, GE Healthcare, WI, USA) with $1.25 \mathrm{~mm}$ slice thickness. The patient heads were immobilized by a thermoplastic mask with a plastic holder placed under the heads. The mask was rigidly attached to the CT couch to ensure setup reproducibility. A customized vacuum bag was made to immobilize the patients' bodies. The same setup was used through simulation and treatment. The patients were sent for an MRI scan (Magnetom, Siemens Healthcare, PA, USA) after their planning CT scan in the same day. IV contrast was administered to every patient and T1-weighted MRI scans were acquired with a
Magnetization-Prepared Rapid Gradient-Echo (MP-RAGE) imaging technique with $1 \mathrm{~mm}$ slice thickness.

\begin{tabular}{|l|l|}
\hline Targets & 35 \\
\hline Patients & 25 \\
\hline Primary tumors & 16 \\
\hline Metastasis tumors & 19 \\
\hline Lung & 8 \\
\hline Breast & 9 \\
\hline Other sites & 2 \\
\hline GTV volumes & $11.3 \pm 10.6 \mathrm{cc}$ \\
\hline Size & $3.4 \pm 1.5 \mathrm{~cm}$ \\
\hline SRT treatment fraction & $4.3 \pm 1.5$ \\
\hline SRT dose & $23.0 \pm 5.3 \mathrm{~Gy}$ \\
\hline
\end{tabular}

Table 1: Patient and treatment characteristics.

\section{Skull based fusion}

The planning CT and MRI with contrast images were first sent to the Treatment Planning System (TPS) (Multiplan 3.5.2, Accuray, CA, USA) and skull based fusion was performed. The planning CT and MRI were chosen as the reference and floating images, respectively. Three to five pairs of control points were manually picked from both images. The rigid displacements derived from the pairs of control points were applied to the MRI images as the initial transformation. An intensity-based image registration was then started by using Mutual Information (MI) as the metric for evaluating the similarities between the two sets of images. The algorithm iteratively found transformations to maximize MI until the images converged to the highest possible match. After the semi-automatic fusions were completed, the fused MRI images were overlain on the planning CT images, and the skull and bony structures from both images were reviewed in the axial, sagittal, and coronal planes. If any mismatches were found, fine-tuning was then manually performed by the physicists (Q.X. and Y.Z.) to reach the best skull-based fusion.

\section{Soft-tissue based fusion}

The planning CT and fused MRI images from the previous step were exported to another commercial software (VelocityAI, Velocity Medical Solutions, GA, USA) for soft-tissue fusion. A physicist (Y.Z.) started by examining the regions near the tumors in the planning CT images and discerned any nearby visible soft-tissue such as gyri, sulci, fissures, and the tumor itself. The alignment of the soft-tissue and tumors between the planning CT and fused MRI images was reviewed. If a mismatch was found, subsequent fine-tuning was performed by manually aligning those soft-tissue features. To reduce inter-observer variations, another physicist (Q.X.) reviewed the manual fine-tuning performed by Y.Z. If there was disagreement, additional fine-tuning, if necessary, was performed until both physicists agreed upon the final soft-tissue based fusion. The final fusions were reviewed and approved by the oncologist (G.K.). The reported translational and rotational shifts after soft-tissue based fusion indicated displacements of softtissue relative to the skull during the patient's CT-to-MRI fusion. If a 
patient had multiple lesions, the same fine-tuning process was repeated for each lesion.

\section{Margin study}

A margin study was performed to assess the GTV coverage since such potential displacements could also carry over into the image guided treatment based on skull positioning. For each patient, the GTV was delineated based on the MRI images after the skull-based fusion. The PTV was generated by adding a $2 \mathrm{~mm}$ margin in all three directions around the GTV. To simulate the tumor displacements relative to skull matching, the GTV was then translated and rotated based on the transformation derived from the soft-tissue based fusion. The transformed GTV was overlain on the PTV contour and its coverage was evaluated.

\section{Results}

There were 25 patients involved in this study, with a total of 35 legions that were compared (Table 1). In 5 out of the 35 lesions, no fine-tuning was needed since the soft-tissue and tumors were aligned well after the skull based fusions. For 24 lesions, minor mismatch between the soft-tissue and skull based fusions was found. The corresponding mean translational and rotational shifts and standard deviations were $0.05 \pm 0.63 \mathrm{~mm}, 0.01 \pm 0.79 \mathrm{~mm}, 0.37 \pm 1.01 \mathrm{~mm}$ in the $\mathrm{x}(\mathrm{LR}), \mathrm{y}(\mathrm{AP})$, and $\mathrm{z}(\mathrm{SI})$ directions, respectively, and $-0.15 \pm 0.67^{\circ}$, $-0.19 \pm 0.34^{\circ}$, and $-0.12 \pm 0.49^{\circ}$ in the pitch, yaw, and roll directions, respectively. In the remaining 6 lesions, relatively large translational and rotational shifts were observed between the two fusions. For those lesions, the translational and rotational shifts were $0.90 \pm 2.15 \mathrm{~mm}$, $1.50 \pm 2.27 \mathrm{~mm}$, and $-1.01 \pm 1.83 \mathrm{~mm}$ in $\mathrm{x}, \mathrm{y}$, and $\mathrm{z}$ directions, respectively, and $-1.42 \pm 3.12^{\circ}, 0.02 \pm 0.83^{\circ}$, and $-0.17 \pm 0.68^{\circ}$ in the pitch, yaw, and roll directions, respectively. The mean $3 \mathrm{D}$ translational shift for all patients was $1.58 \pm 1.37 \mathrm{~mm}$.

Three cases are displayed in Figures 1-4. The first case was an outlier patient with the greatest mismatch between skull based and soft-tissues based fusions. The patient had a metastatic brain lesion, originating from a small-cell lung carcinoma, located at the gray-white matter junction. The translational and rotational shifts between the two fusions were $2.03 \mathrm{~mm}, 4.16 \mathrm{~mm}$, and $-0.68 \mathrm{~mm}$ in the $\mathrm{x}, \mathrm{y}$, and $\mathrm{z}$ directions, respectively, and $-7.02^{\circ}, 1.01^{\circ}$, and $-0.96^{\circ}$ in the pitch, yaw, and roll directions, respectively. We first reviewed the skull and bony structure matching between the two fusions. After skull-based fusion (Figure 1a) the skull and bony anatomy matched well in the sagittal plane. After soft-tissue based fusion (Figure 1b), a large pitch or head up/down rotational shift was observed, as the nose did not match well in the overlay of the CT and MR images. For this patient, we further reviewed the soft-tissue matching between the two fusions after zooming in.

In Figure 2, the axial (2a) and sagittal (2b) slices of the CT and MR images for the outlier patient are displayed, with CT on top, MR after skull based fusion in the middle, and MR after soft-tissue based fusion on the bottom. The crosshairs indicate the center of the lesion after fusion. The lesion was somewhat discernible in the planning CT, as can be observed in the same region of the MR images. After skull based fusion, large deviations in the lesion and soft-tissue were observed between CT and MRI in the axial plane, as shown in the top and middle images of Figure 2a. After soft-tissue based fusion, the textures of the gyri and fissures matched well in the axial plane, as shown in the top and bottom images of Figure 2a.

Similar deviations between the textures after skull based fusion are shown in the sagittal plane in the top and middle images of Figure $2 \mathrm{~b}$, and similar agreement between textures after soft-tissue based fusion are shown in the top and bottom images of Figure $2 \mathrm{~b}$. The same rotational shift in pitch that is observed in Figure 1 can also be found in Figure 2b. The second patient, shown in Figure 3 (top: axial, bottom: coronal), had a recurrent meningioma in the left frontal and parietal lobes.

The fused MRI with contrast (inside the region of interest box) was overlaid with the planning CT. The lesion (near the midline), and the nearby visible gyrus and fissure were contoured in the planning CT. After skull based fusion (Figure 3a), the contours from the planning CT slightly deviated from the soft-tissue in the fused MR images. After soft-tissue based fusion (Figure $3 \mathrm{~b}$ ), the matching between the softtissue and lesions were improved. The third patient, shown in Figure 4, had surgical resection and previous irradiation of a meningioma in the left frontal and parietal lobes. Abnormal enhancement was observed in the MR images in the region around the craniotomy, possibly due to edema and swelling associated with the meningioma.

The region around the craniotomy was outlined in the planning CT and overlaid with the MR images. After skull based fusion (Figure 4a), the region around the craniotomy showed slight mismatch, and overlapping with the peripheral abnormal enhancement was observed. After soft-tissue based fine-tuning (Figure 4b), the alignment of the region around the craniotomy in CT and MRI was improved. We also observed slight mismatch of the skull after soft-tissue based fusion (bottom of Figure $4 \mathrm{~b}$ ), indicating relative displacement between the two fusions.

We further performed a margin study to investigate if the mismatch between the two fusions could be accounted for with a small margin. For 29 lesions with minor to no mismatch, the GTV was $100 \%$ covered by a PTV that was created using a $2 \mathrm{~mm}$ margin. For the outlier patient in Figures 1 and 2, after soft-tissue based fusion nearly the entire GTV was not covered by the PTV due to large discrepancy between the two fusions and the small size of the lesion $(<1 \mathrm{cc})$. In the remaining 5 lesions, we found after soft-tissue based fusions that on average $98.9 \%$ of the GTV targets were encompassed by the PTV using a $2 \mathrm{~mm}$ margin.

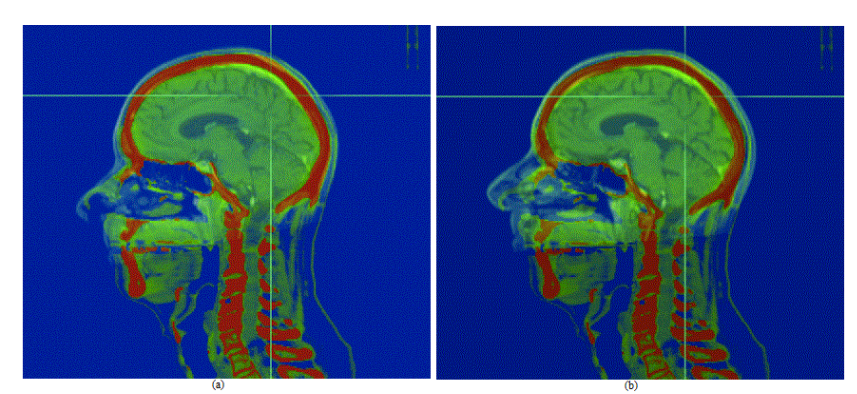

Figure 1: Illustration of a large pitch angle between the skull-based (a) and soft-tissue-based (b) fusion for patient 1. 


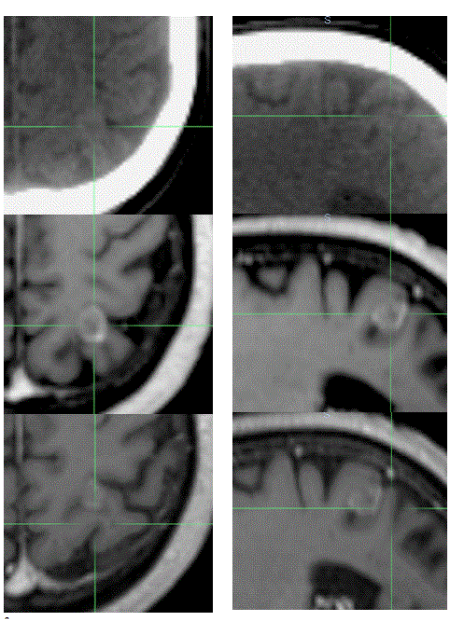

Figure 2: Skull and soft-tissue fusion comparison for patient lin the axial (a) and sagittal (b) directions (top: planning CT, middle: softtissue-based fusion, bottom: skull-based fusion).

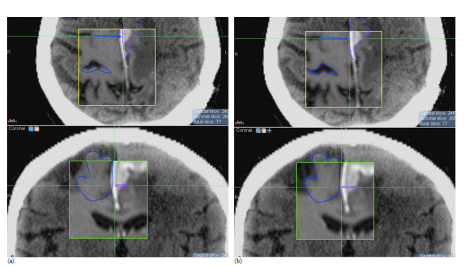

Figure 3: Skull (a) and soft-tissue (b) based fusion comparison for patient 2, with the axial slice on top and the coronal slice at the bottom.

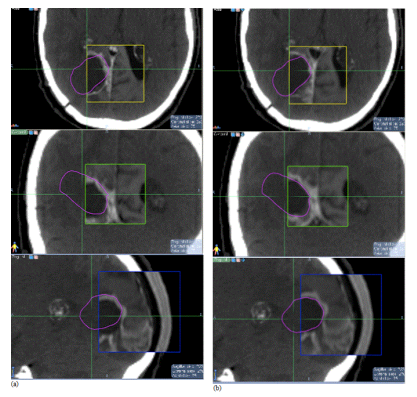

Figure 4: Skull (a) and soft-tissue (b) based fusion comparison for patient 3, with the axial slice on top, the coronal slice in the middle, and the sagittal slice at the bottom.

\section{Discussion}

A study by Guckenberger et al. evaluated the reliability of the bony anatomy guidance for SRS of brain metastases. They concluded that the skull was a sufficient surrogate for LINAC-based SRS of brain metastases. For 20 brain metastases analyzed in their study, they reported displacements (mean: $1.7 \pm 0.7 \mathrm{~mm}$, maximum: $2.8 \mathrm{~mm}$ ) between fusions based on the skull and soft-tissue. Currently, MRI, with its high soft-tissue contrast, is the best available imaging modality for tumor delineation and soft-tissue visualization in the brain. The displacements observed in our CT-MRI study (mean: $1.58 \pm 1.37 \mathrm{~mm}$ ) were very close to those reported by Guckenberger et al. aside from an outlier patient. In addition to the translational displacements, we further reported rotation angles between the two fusions in pitch, yaw, and roll. Although the magnitude of the displacement is relatively small, we hold that special attention is needed for soft-tissue differences since such displacements are highly patient specific, skull based localization during treatment could carry over such uncertainties, and deviations could ultimately lead to under-dosing the lesion or even target miss in special cases. An important aspect of this is that CT scan performed without contrast will not be able to visualize soft tissues and thus if there is a significant mis-alignment this will not be noted. For this reason both the planning CT scan and the MRI should be performed with contrast in order to evaluate for

The dosimetric effects of the displacements are not the focus of this study. However, a recent study by Zhang et al. stated that dosimetric uncertainties due to setup were associated with the location of the brain tumor [16]. In their margin study, dosimetric coverage of the Clinical Target Volume (CTV) was evaluated based on the isocenter shift derived after matching the CBCT and the planning CT. They found that the greatest under-dosing occurred in an outlier lesion located in the posterior brain, due to the unique geometry of the posterior head. A margin greater than $3 \mathrm{~mm}$ was needed to ensure a high percentage of coverage to the CTV. Interestingly, the outlier lesion in our study was also located in the same area of the brain. The coincidence suggests more geometric uncertainties may be associated with brain tumors in the posterior brain. This would raise more concerns for CyberKnife based SRS and SRT since only the anterior and superior part of the skull is used as the matching anatomy during treatment. However, this needs to be further investigated using more clinical data in future studies.

We performed a margin study to evaluate the GTV coverage if displacements occurred. All lesions except for the outlier were covered well after adding a $2 \mathrm{~mm}$ margin. Whether to add a margin to brain tumors or not during SRS and SRT, however, is still controversial and debatable in the community. Various mathematical models and formulas have been proposed in the literature to determine the size of margin to be added [17-21]. There is no unified method used to determine the size of the margin needed to cover all treatment uncertainties. In current practice, the size of the margin is mostly determined based on the physician's clinical experience [22]. In our study, we found a $2 \mathrm{~mm}$ margin should be sufficient to accommodate for displacements since all of the lesions, except for the outlier, were covered well by the PTV. Note, however, that it may not be necessary to apply a margin to all patients since noticeable displacement was found in only $17 \%$ of the patients enrolled in the study. We suggest that the decision should be made based on a combination of the physician's clinical experience, review of soft-tissue and tumor matching, the size of the tumor, and possibly the location of the tumor.

The actual causes of the relative displacements between the softtissue and skull based fusions are still not clear. A study by Monea et al. investigated the role of relative brain-skull motion in the etiology of traumatic brain injury. Under quasi-static circumstances, 30 healthy volunteers had MRI scans in 4 different head positions. Image registration between these MR images revealed complex brain motion patterns, which were very regional and subject dependent. For 3 
groups of volunteers categorized by age, the $3 \mathrm{D}$ sagittal brain deviations were $-0.25 \pm 1.62 \mathrm{~mm}$ in $\mathrm{x},-0.47 \pm 1.90 \mathrm{~mm}$ in $\mathrm{y}$, and -0.16 $\pm 1.19 \mathrm{~mm}$ in $\mathrm{z}$, and $3 \mathrm{D}$ coronal brain deviations were $-0.66 \pm 2.33 \mathrm{~mm}$ in $\mathrm{x},-0.23 \pm 1.35 \mathrm{~mm}$ in $\mathrm{y}$, and $-0.05 \pm 1.52 \mathrm{~mm}$ in $\mathrm{z}$ with a $95 \%$ confidence level. The authors stated that multiple factors contributed to the relative brain-skull motion, including gravity, contact surfaces, fluid pressure and material properties. Unlike the experiment designed by Monea et al. great efforts have been made to ensure reproducibility of head position during SRS and SRT. However, relative displacement could still exist between simulation and treatment due to setup variation, internal pressure, and property changes. For example, in our study the couch of the MRI scanner was not flat. During the MRI scan, a standard "curved" diagnostic couch was used and radiofrequency (RF) coils were placed on the couch. The same technologist who performed the CT scan adjusted the height of the head holder and the layers of the blanket underneath the patient to best reproduce the same head position for the MRI scan. Slight setup variations during the MRI scan could still occur, which has been a common problem for the majority of clinics. Designing of a flat couch for an MRI scanner is still a research topic [23], and designated MRI scanners with a flat couch are only available in a few research institutions. In the CT-to-CT study by Guckenberger et al. this factor was excluded since all the patients were scanned on a flat couch with a thermal mask and body immobilization devices, but soft-tissue and skull based fusion displacements were still observed. Thus, causes for displacement could be due to minor setup variations, slight tumor motion, or even using simple skull geometries to characterize the complicated arrangement of soft-tissue and tumors within the brain.

There are potentially also several sources of error within the current study. First, fusion errors could be induced during the skull based and soft-tissue based fusions. However, for skull based fusion, studies have demonstrated that sub-millimeter accuracy was achievable for rigid registration of the skull in CT and MR images using MI [24,25]. Also, for soft-tissue based fusion, to reduce inter-observer variations, fusions were reviewed and fine-tuned until the best match could be agreed upon between two physicists. The final fusion was also then reviewed and approved by the radiation oncologist (G.K). It is important to note that patients selected for this study had targets that were able to be visualized on the CT scan, CT scan without contrast are less likely to have visible targets. Second, MR imaging suffers from inherent distortions due to in homogeneities in the magnetic field, nonlinearities in the gradient of the magnetic field, view angles, susceptibility to chemical shift variations, etc. With proper imaging protocols, calibration of the scanner, and correction techniques, the distortions can be minimized and sub-voxel accuracy could be achieved. Lastly, the relative displacement reported in this study was between CT and MRI scans that were performed on the same day. This may not represent the actual displacement variations at the time of treatment since the treatment is often a few days after the scans.

\section{Conclusion}

Our study shows noticeable variations between skull based and softtissue based fusion in $17 \%$ of the observed lesions with typical patient setup for CT and MRI scans. For accurate target localization, skullbased fusions should be reviewed and fine-tuned under nearby softtissue guidance, and if visible in both images, tumor guidance as well. Both the CT and MRI scans should be performed with contrast to ensure adequate target visualization and to determine if soft tissue adjustment is required. If substantial deviations are found between fusions, a margin may be useful to ensure target coverage during treatment.

\section{References}

1. Leksell L (1951) The stereotaxic method and radiosurgery of the brain. Acta Chir Scand 102: 316-319.

2. Masi L, Casamassima F, Polli C, Menichelli C, Bonucci I, et al. (2008) Cone beam CT image guidance for intracranial stereotactic treatments: comparison with a frame guided set-up. Int J Radiat Oncol Biol Phys 71: 926-933.

3. Pommier P, Gassa F, Lafay F, Claude L (2009) Image guided radiotherapy with the Cone Beam CT kV (Elekta): experience of the Leon Berard centre. Cancer radiotherapie: journal de la Societe francaise de radiotherapie oncologique 13: 384-390.

4. Lightstone AW, Tsao M, Baran PS, Chan G, Pang G, et al. (2012) Cone beam CT (CBCT) evaluation of inter-and intra-fraction motion for patients undergoing brain radiotherapy immobilized using a commercial thermoplastic mask on a robotic couch. Technol Cancer Res Treat 11: 203-209.

5. Dhabaan A, Schreibmann E, Siddiqi A, Elder E, Fox T, et al. (2012) Six degrees of freedom CBCT-based positioning for intracranial targets treated with frameless stereotactic radiosurgery. Journal of Applied Clinical Medical Physics: 13.

6. Peng JL, Kahler D, Li JG, Samant S, Yan G, et al. (2010) Characterization of a real-time surface image-guided stereotactic positioning system. Med Phys 37: 5421-5433.

7. Li G, Ballangrud A, Kuo LC, Kang H, Kirov A, et al. (2011) Motion monitoring for cranial frameless stereotactic radiosurgery using videobased three-dimensional optical surface imaging. Med Phys 38: 3981.

8. Keshavarzi S, Meltzer H, Ben-Haim S, Newman CB, Lawson JD, (2009) et al. Initial clinical experience with frameless optically guided stereotactic radiosurgery/radiotherapy in pediatric patients. Childs Nerv Syst 25: 837-844.

9. Adler JR Jr, Chang SD, Murphy MJ, Doty J, Geis P, et al. (1997) The Cyberknife: a frameless robotic system for radiosurgery. Stereotact Funct Neurosurg 69: 124-128.

10. Ma J, Chang Z, Wang Z, Jackie Wu Q, Kirkpatrick JP, et al. (2009) ExacTrac $X$-ray 6 degree-of-freedom image-guidance for intracranial non-invasive stereotactic radiotherapy: comparison with kilo-voltage cone-beam CT. Radiother Oncol 93: 602-608.

11. Murphy MJ (1997) An automatic six-degree-of-freedom image registration algorithm for image-guided frameless stereotaxic radiosurgery. Med Phys 24: 857-866.

12. Takeuchi H, Yoshida M, Kubota T, Ishii H, Sato K, et al. (2003) Frameless stereotactic radiosurgery with mobile $\mathrm{CT}$, mask immobilization and micro-multileaf collimators. Minim Invasive Neurosurg 46: 82-85.

13. Guckenberger M, Baier K, Guenther I, Richter A, Wilbert J, et al. (2007) Reliability of the bony anatomy in image-guided stereotactic radiotherapy of brain metastases. Int J Radiat Oncol Biol Phys 69: 294-301.

14. Feng Y, Abney TM, Okamoto RJ, Pless RB, Genin GM, et al. (21010) Relative brain displacement and deformation during constrained mild frontal head impact. J R Soc Interface 7: 1677-1688.

15. Monea AG, Verpoest I, Vander Sloten J, Van der Perre G, Goffin J, et al. (2012) Assessment of relative brain-skull motion in quasistatic circumstances by magnetic resonance imaging. J Neurotrauma 29: 2305-2317.

16. Zhang Q, Chan MF, Burman C, Song Y, Zhang M (2013) Three independent one-dimensional margins for single-fraction frameless stereotactic radiosurgery brain cases using CBCT. Med Phys 40: 121715 .

17. van Herk M (2004) Errors and margins in radiotherapy. Semin Radiat Oncol 14: 52-64.

18. Jones D (1994) ICRU Report 50óPrescribing, recording and reporting photon beam therapy. Med Phys 21: 833. 
Citation: Xu Q, Hanna G, Zhai Y, Asbell S, Fan J, et al. (2015) Assessment of Brain Tumor Displacements after Skull-based Registration: A CT/MRI Fusion Study. J Nucl Med Radiat Ther 6: 265. doi:10.4172/2155-9619.1000265

Page 6 of 6

19. Mageras GS, Fuks Z, Leibel SA, Ling CC, Zelefsky MJ, et al. (1999) Computerized design of target margins for treatment uncertainties in conformal radiotherapy Int J Radiat Oncol Biol Phys 43: 437-445.

20. Yan D, Lockman D, Martinez A, Wong J, Brabbins D, et al. (2005) Computed tomography guided management of interfractional patient variation. Semin Radiat Oncol 15: 168-179.

21. van Herk M, Remeijer P, Rasch C, Lebesque JV (2000) The probability of correct target dosage: dose-population histograms for deriving treatment mlargins in radiotherapy. Int J Radiat Oncol Biol Phys 47: 1121-1135.

22. Purdy JA (2004) Current ICRU definitions of volumes: limitations and future directions. Semin Radiat Oncol 14: 27-40.
23. McJury M, O'Neill A, Lawson M, McGrath C, Grey A, et al. (2011) Assessing the image quality of pelvic $\mathrm{MR}$ images acquired with a flat couch for radiotherapy treatment planning. Br J Radiol 84: 750-755.

24. Maes F, Collignon A, Vandermeulen D, Marchal G, Suetens P (1997) Multimodality image registration by maximization of mutual information. IEEE Trans Med Imaging 16: 187-198.

25. West J, Fitzpatrick JM, Wang MY, Dawant BM, Maurer CR Jr, et al. (1997) Comparison and evaluation of retrospective intermodality brain image registration techniques. J Comput Assist Tomogr 21: 554-566. 